\title{
Delayed Mesoderm and Erythroid Differentiation of Murine Embryonic Stem Cells in the Absence of the Transcriptional Regulator FUBP1
}

\author{
Josephine Wesely, ${ }^{1}$ Marlene Steiner, ${ }^{1}$ Frank Schnütgen, ${ }^{2}$ Manuel Kaulich, ${ }^{3}$ \\ Michael A. Rieger, ${ }^{2,4}$ and Martin Zörnig ${ }^{1,4}$ \\ ${ }^{1}$ Georg-Speyer-Haus, Institute for Tumor Biology and Experimental Therapy, Paul-Ehrlich-Strasse 42-44, \\ 60596 Frankfurt/Main, Germany \\ ${ }^{2}$ LOEWE Center for Cell and Gene Therapy Frankfurt and Department for Medicine, Hematology/Oncology, Goethe University \\ Hospital Frankfurt/Main, 60590 Frankfurt/Main, Germany \\ ${ }^{3}$ Institute of Biochemistry II, Goethe University Frankfurt, 60590 Frankfurt/Main, Germany \\ ${ }^{4}$ German Cancer Consortium (DKTK), 69120 Heidelberg, Germany
}

Correspondence should be addressed to Martin Zörnig; zoernig@gsh.uni-frankfurt.de

Received 21 December 2016; Revised 2 March 2017; Accepted 19 March 2017; Published 15 May 2017

Academic Editor: Zhaohui Ye

Copyright (C) 2017 Josephine Wesely et al. This is an open access article distributed under the Creative Commons Attribution License, which permits unrestricted use, distribution, and reproduction in any medium, provided the original work is properly cited.

\begin{abstract}
The transcriptional regulator far upstream binding protein 1 (FUBP1) is essential for fetal and adult hematopoietic stem cell (HSC) self-renewal, and the constitutive absence of FUBP1 activity during early development leads to embryonic lethality in homozygous mutant mice. To investigate the role of FUBP1 in murine embryonic stem cells (ESCs) and in particular during differentiation into hematopoietic lineages, we generated Fubp1 knockout (KO) ESC clones using CRISPR/Cas9 technology. Although FUBP1 is expressed in undifferentiated ESCs and during spontaneous differentiation following aggregation into embryoid bodies (EBs), absence of FUBP1 did not affect ESC maintenance. Interestingly, we observed a delayed differentiation of FUBP1-deficient ESCs into the mesoderm germ layer, as indicated by impaired expression of several mesoderm markers including Brachyury at an early time point of ESC differentiation upon aggregation to EBs. Coculture experiments with OP9 cells in the presence of erythropoietin revealed a diminished differentiation capacity of Fubp1 KO ESCs into the erythroid lineage. Our data showed that FUBP1 is important for the onset of mesoderm differentiation and maturation of hematopoietic progenitor cells into the erythroid lineage, a finding that is supported by the phenotype of FUBP1-deficient mice.
\end{abstract}

\section{Introduction}

The far upstream element (FUSE) binding protein 1 (FUBP1) was identified as a transcriptional regulator that binds to the single-stranded AT-rich FUSE DNA sequence $1.5 \mathrm{~kb}$ upstream of the $c-m y c$ promoter [1]. We and others found FUBP1 to be upregulated in a number of tumor entities, such as hepatocellular carcinoma (HCC), prostate, and colorectal cancer [2-5]. Our studies demonstrated an essential role for FUBP1 in HCC tumorigenesis and established FUBP1 as a pro-proliferative and antiapoptotic oncoprotein [4].
In our recent work, we analyzed the physiological role of FUBP1 in two independent functional FUBP1 knockout mouse models. In both models, FUBP1 deficiency led to embryonic lethality around day E15.5 and a strong anemic phenotype [6]. The embryos displayed a reduced number of hematopoietic stem cells (HSCs) in the fetal liver, and in contrast to wildtype controls, the remaining FUBP1deficient HSCs were not able to repopulate the blood lineages in a competitive transplantation experiment. Our studies established FUBP1 as an important regulator of HSC self-renewal. In addition, we noticed that the ery- 
throid lineage in the FUBP1 mutant E15.5 embryos showed a diminished proportion of mature cells, hinting towards an erythroid differentiation defect in the absence of FUBP1 [6].

The essential role of FUBP1 in HSC self-renewal raises the question about the potential role of the protein in other stem cells. Interestingly, the pathohistological analysis of Fubp1 knockout embryos showed abnormalities during the development of the placenta and of lymphoid tissue and an increased parenchymal cellularity in the brain [7]. Embryonic stem cells (ESCs) are pluripotent cells, that is, they possess an infinite self-renewal potential and can differentiate into cells of all three germ layers (ectoderm, endoderm, and mesoderm) and the germline, ultimately contributing to all lineages of the mature organism [8]. Since the 1980s, mouse ESCs can be isolated from the inner cell mass of blastocysts (most suitable at day $\mathrm{E}$ 3.5) and cultivated on feeder cells, which usually consist of replication-deficient fibroblasts. Addition of leukemia inhibitory factor (LIF) to the growth medium can substitute the feeder cells, and ESC lines cultured on gelatincoated plates in the presence of LIF still maintain their stemness $[9,10]$. The recent progress in the ESC research field holds high promise for biomedicine and transplantation medicine as well as for the pharmaceutic developmental research [11, 12]. Discovering novel genes important for specific differentiation decisions led to huge efforts to employ ESCs for cellular therapies [13].

A number of protocols for the differentiation of ESCs into a variety of cell types were established in the last two decades of stem cell research [14-16] (for review of literature describing specifically the in vitro differentiation of ESCs towards the hematopoietic lineage see for example $[17,18])$. However, the formation of EBs, which represents the early embryonic development, is a spontaneous germ layer differentiation induced by the absence of LIF and used in almost every differentiation protocol as a first step $[19,20]$. The embryonic stem cells undergo a rapid differentiation process during the formation of EBs, and the stem cell markers such as Oct4 and Nanog are downregulated. In parallel, a rapid upregulation of markers for the three germ layers ectoderm, endoderm, and mesoderm occurs [21].

The aim of this study was to analyze the function of FUBP1 in murine embryonic stem cells during spontaneous differentiation upon aggregation to EBs in the absence of LIF. In addition, we wanted to employ the induction of erythropoiesis in ESCs as a suitable cell culture model to complement our in vivo studies on the role of FUBP1 during erythropoiesis in FUBP1-deficient mice [6]. We established Fubp1 knockout ESC clones with the help of the CRISPR/Cas9 technology [22] and analyzed the consequences of FUBP1 deficiency in ESCs and during EB formation using the stem cell markers Oct4 and Nanog [23-26] and a number of differentiation markers indicative for the mesoderm, ectoderm, and endoderm germ layer cells. Finally, we cocultured the ESCs with OP9 cells $[27,28]$ to study the direct effect of FUBP1 inactivity for erythroid differentiation.

\section{Materials and Methods}

2.1. Cell Lines Used, Embryonic Stem Cell Culture, EB Differentiation, and Erythroid Differentiation. The mouse ESC line E14TG2A [29] was cultured on 0.1\% gelatincoated plates in Glasgow's Minimal Essential Medium (GMEM; Sigma), supplemented with $2 \mathrm{mM}$ glutamine (GIBCO), $1 \mathrm{mM}$ sodium pyruvate (GIBCO), 1x nonessential amino acids (GIBCO), 10\% ES cell-qualified FBS (Thermo Fisher Scientific), $10 \%(v / v)$ of a $1: 1000$ dilution of $\beta$-mercaptoethanol stock solution, and $500-1000 \mathrm{u} / \mathrm{ml}$ of leukocyte inhibitory factor (LIF; Chemicon). ESCs were aggregated to embryoid bodies by plating $10^{4}$ cells/ml on bacteria dishes in medium without LIF to induce spontaneous differentiation. For erythroid differentiation of ESCs, OP9 stroma cells (ATCC CRL-2749) were used as a coculture system in the presence of human SCF (100 ng/ $\mu$; Peprotech) and human EPO (2 U/ml; Roche) as described in [27, 28]. Briefly, OP9 cells were seeded 4 days before adding $1 \times 10^{4}$ ESCs to the confluent OP9 cell layer. At day 5 of the coculture, ESCs were replated in a $1: 200$ dilution on new OP9 cells in the presence of SCF. Additionally, mesoderm (FLK-1) and hemangioblast marker (FLK-1/VE-Cad) were analyzed by flow cytometry. At day 12 of coculturing, hematopoietic cells were identified by using CD45 as a marker. For erythroid differentiation, ESCs were cultured on OP9 cells for 5 days, then SCF and EOP were added (without replating) for additional 5 days, before flow cytometry analysis was performed using CD71 and Ter119 as differentiation markers.

2.2. Generation of Fubp1 Knockout and Nontarget Control Clones Using the CRISPR/CAS9 System. The lentiviral CRISPR/Cas9 vector $p L C V 2 v$ [30] was used to introduce the two gRNAs \#1 (5'-caaaaattgggggtgatgc- $\left.3^{\prime}\right)$ and \#2 $\left(5^{\prime}\right.$ agatgcctgcagagagcg-3') recognizing the first (\#2) and second (\#1) murine Fubp1 exon and one nontarget control (NTC) sequence $\left(5^{\prime}\right.$-ttccgggctaacaagtcct-3'). E14TG2A cells were transduced and selected with $2 \mu \mathrm{g} / \mathrm{ml}$ puromycin to establish single-cell clones. Analysis of outgrowing potentially FUBP1deficient clones was performed by western blot and immunohistochemical staining with anti-FUBP1 antibody.

2.3. RNA Preparation, cDNA Synthesis, and Quantitative Real-Time PCR. RNA was prepared using the RNeasy® Mini Kit (Qiagen) according to the manufacturer's manual. $1 \mu \mathrm{g}$ of total RNA was reverse transcribed using the Omniscript ${ }^{\circledR}$ Reverse Transcription Kit (Qiagen) following the manufacturer's instructions, with additional on-column DNaseI digestion. mRNA expression levels were quantified using a LightCycler480 (Roche) with 96 well plates (4-titude) and SYBR® Select Master Mix (Thermo Fisher Scientific). qPCR reactions were performed in technical duplicates in a total reaction volume of $20 \mu \mathrm{l}$. mRNA levels were either normalized to Gapdh expression and calculated according to the $2^{-}$ ${ }^{\Delta} \Delta \mathrm{Ct}$ method [31] or presented as the relative mRNA expression compared to Gapdh.

2.4. Western Blot Analysis. FUBP1 expression was detected via immunoblot with an antiFUBP1 (1:1000; clone N-15, Santa Cruz), and $\beta$-actin levels were assessed as a loading 


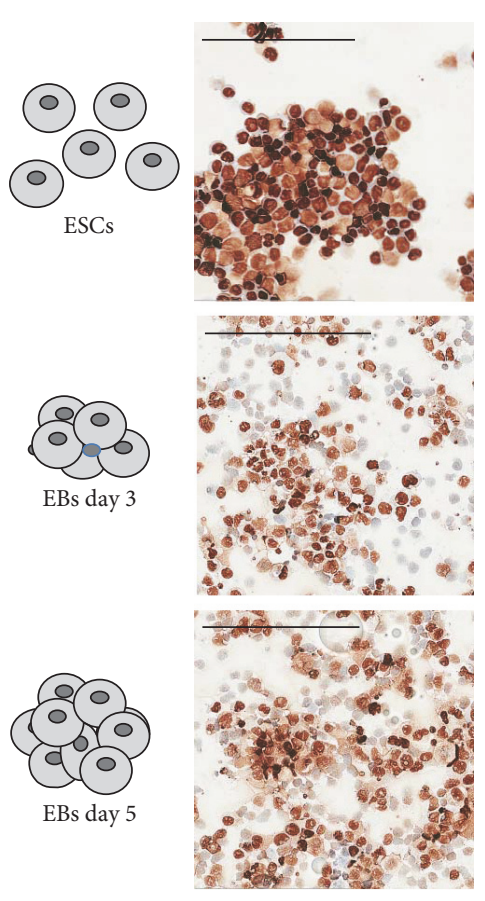

(a)

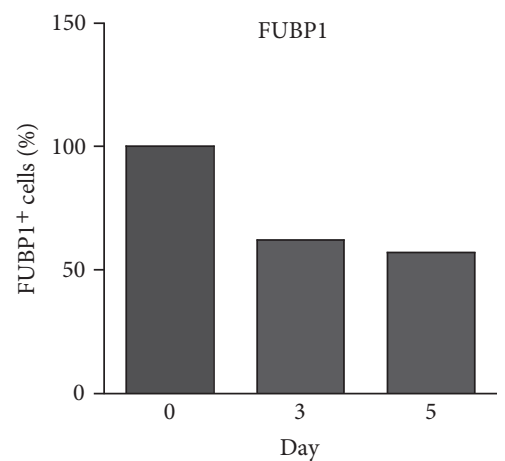

(b)

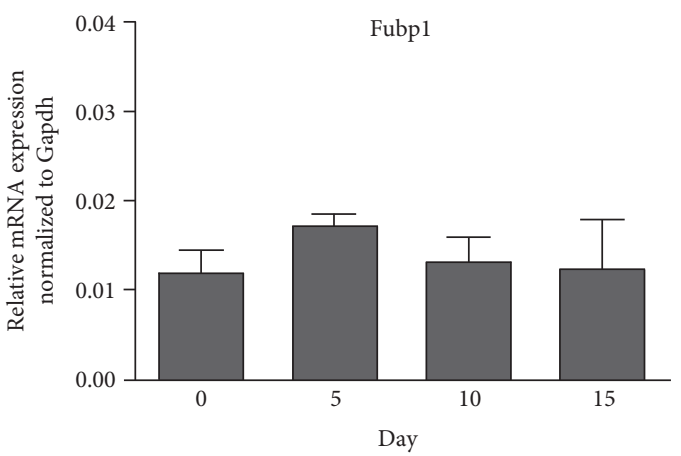

(c)

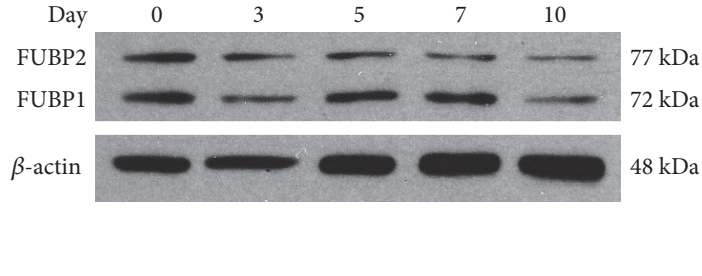

(d)

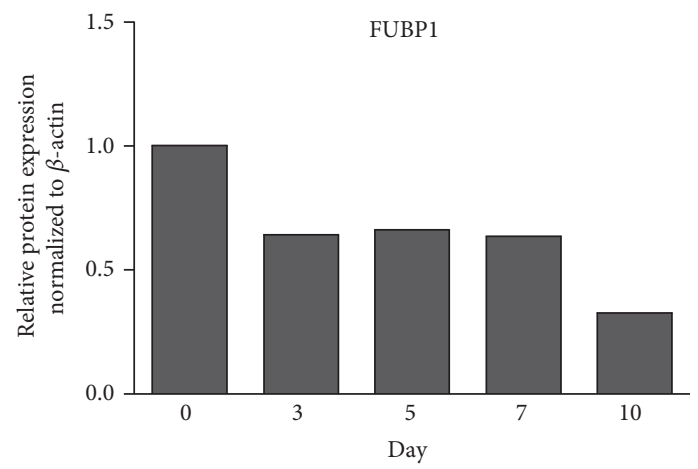

(e)

FIGURE 1: FUBP1 expression in undifferentiated ESCs and during differentiation in EBs. (a) Immunohistochemical (IHC) analysis of ESCs during normal cell culture and dissociated EBs at days 3 and 5 of spontaneous differentiation in the absence of LIF revealed significant but heterogeneous FUBP1 reactivity in the cells. (b) Visual quantification of FUBP1-negative cells based on the IHC analysis shown in (a). Between 267 and 471 cells were counted for each time point, and one slide per time point was analyzed. (c) qPCR analysis of RNA that was isolated from EBs revealed no obvious changes in FUBP1 mRNA expression during differentiation. (d) FUBP1 protein levels in EBs at different time points of spontaneous differentiation as determined by western blotting. (e) represents the quantification of the blot shown in (d). The qPCR data represent the mean values $\pm \mathrm{SD}(n=3)$, $C_{\mathrm{t}}$ values were normalized to Gapdh expression. Scale bars in (a) indicate $200 \mu \mathrm{m}$. 
control using a goat-derived antiserum $(1: 2000$; clone C- 11 , Santa Cruz). As the secondary antibody, a rabbit anti-goat antibody (1:10,000; cat. no. 81-1620; Invitrogen) was chosen for detection. Quantification of FUBP1 western blots was performed using the FUSION Fx system (Vilber Lourmat).

2.5. Immunohistochemistry. ESCs were trypsinized, and EBs were dissociated with $500 \mu \mathrm{l}$ Accutase (Sigma). After washing with PBS, 50,000 cells were resuspended in $70 \mu \mathrm{l}$ PBS and spun onto a polysine adhesion slide (Thermo Fisher Scientific) with a Cellspin II centrifuge (Tharmac). Staining was performed as follows: EtOH solution $\left(10 \mathrm{~min} ; 100^{\circ} \mathrm{C}\right)$, BondTM Wash ( $3 \mathrm{~min}$; RT), Peroxid (10 min; RT), antiFUBP1 (abcam 181,111; 30 min; RT), Polymer (8 min; RT), BondTM Wash (4 min; RT), DAB (3,3'-diaminobenzidine; 8 min; RT), Hematoxylin (10 min; RT), and $\mathrm{H}_{2} \mathrm{O}$ (1 min; RT).

2.6. Flow Cytometry. EBs were dissociated with $500 \mu \mathrm{l}$ Accutase solution (Sigma). After washing with PBS, the single cells were stained with fixable viability dye APC-eF780 (eBioscience), anti-CD309 (FLK-1/VEGFR2)-PE (BioLegend), and anti-mouse CD45.2 PerCP-Cyanine5.5 (eBioscience). Alternatively, cells were fixed, permeabilized, and blocked with mouse IgG before staining with PE-conjugated antimouse Brachyury antibody (R\&D Systems) and corresponding PE-conjugated goat IgG isotype control (R\&D Systems). Erythroid cells obtained from ESCs cocultured with OP9 cells were collected from the supernatant, washed with PBS, and stained with fixable viability dye APC-eF780, anti-CD71APC (eBioscience), and anti-Ter119-PE (eBioscience). Undifferentiated ESCs were trypsinized, washed with PBS, and stained with viability dye APC-eF780, anti-SSEA1-V450 (clone MC480; BD Biosciences), and anti-SSEA4-PE (eBioMC-813-70; eBioscience). For cell cycle analysis, EBs were stained with anti-CD309-PE as described above. Subsequently, cells were washed with PBS and permeabilized in $100 \mu \mathrm{l}$ Cytofix/Cytperm (BD Biosciences). Cells were stained with anti-Ki-67-APC (clone 16A8; BioLegend) overnight. Hoechst 33342 (ImmunoChemistry Technologies) was added immediately before FACS analysis. Flow cytometry was performed with a FACSFortessa (Becton Dickinson), and for data analysis, the FACS Diva software (Becton Dickinson) or FlowJo $^{\circledR}$ was used.

2.7. Statistical Analysis. Statistical analysis was performed with GraphPad Prism software (GraphPad Software), applying the two-tailed $t$-test. $p$ values $<0.05$ were considered statistically significant $(p<0.05: *, p<0.01: * *$, $p<0.001: * * *)$.

\section{Results}

3.1. FUBP1 Is Expressed in ESCs and during EB Differentiation. Undifferentiated ESCs cultured in the presence of LIF and ESCs that were aggregated to EBs for spontaneous differentiation upon plating onto bacterial dishes and removal of LIF were analyzed for the expression of FUBP1. Immunohistochemically (IHC) staining revealed a significant but heterogeneous FUBP1 expression in undifferentiated ESCs on singlecell level (Figure 1(a)). According to our analysis, 38\% of all
ESCs became FUBP1-negative after 3 days of spontaneous differentiation in EBs (Figure 1(b)). While qPCR experiments (Figure 1(c)) revealed no obvious differences in Fubp1 mRNA during spontaneous differentiation in EBs, quantification of western blot results (Figures 1(d) and 1(e)) demonstrated a noticeable downregulation of FUBP1 protein levels from day 3 on of EB culture in the absence of LIF, which is in line with the increased amount of FUBP1-negative cells observed by immunohistochemistry (Figures 1(a) and 1(b)).

\subsection{FUBP1 Expression Is Not Required in Undifferentiated} Murine ESCs for Normal Cell Cycle Progression. To investigate the role of FUBP1 in ESC expansion and during differentiation, the lentiviral CRISPR/Cas9 vector $p L C V 2 v$ was used to introduce one of two Fubp1 gRNAs or a nontarget control sequence (Figure 2(a)). Following puromycin selection of the transduced ESCs, outgrowing clones were isolated and analyzed for FUBP1 protein expression. We could successfully generate 3 Fubp1 knockout clones each, either transduced with gRNA1 or gRNA2, that showed no FUBP1 expression in western blot analysis (Figure 2(b); gRNA1 \#7, $\# 8$, \#14; gRNA2 \#5, \#7, and \#8; for two of the Fubp1 knockout clones, the sequence of the targeted locus (exon 2) is shown in Figure S1 in Supplementary Material available online at https://doi.org/10.1155/2017/5762301; the imperfect repair following the CRISPR/Cas9 manipulation led to a change in the reading frame and the generation of premature stop codons in both alleles of both knockout clones). ESC clones that had been transduced with the nontarget control (NTC) gRNA exhibited a comparable FUBP1 protein expression level as detected for untransduced ESCs. Additionally, the 6 Fubp1 knockout and 3 of the NTC clones were analyzed by immunohistochemistry to confirm the absence of FUBP1 in the knockout clones (Figure 2(c)). No changes in FUBP2 protein expression could be detected in the Fubp1 knockout or NTC ESC clones. For all further investigations, at least three independent experiments, each with the mean values obtained from several Fubp1 knockout and NTC ESC clones (as indicated), were performed.

First, we wanted to confirm that both, Fubp1 knockout and NTC control ESC clones, maintained the same level of pluripotency. Undifferentiated pluripotent mouse ESCs express stage-specific embryonic antigen 1 (SSEA1) which is downregulated during ESC differentiation [32]. In contrast, undifferentiated murine ESCs do not express SSEA4, while their differentiation is accompanied by an increase in SSEA4 expression [33]. Flow cytometry analysis of our ESC clones confirmed their SSEA $1^{+} / \mathrm{SSEA}^{-}{ }^{-}$undifferentiated status (see Figure S2), suggesting that the absence of FUBP1 per se does not interfere with murine ESC pluripotency.

The analysis of Fubp1 knockout and NTC ESC clones showed no significant differences in their cell cycle distribution, and no increase in the number of dead cells could be detected in the absence of FUBP1 (Figure S3A). When we investigated the expression of the two bona fide FUBP1 target genes $p 21$ and $c-m y c$ in Fubp 1 knockout and NTC ESC clones, we noticed no difference in p21 mRNA levels but a significant upregulation of $c-m y c$ in the Fubp1 knockout clones (Figure S3B). 


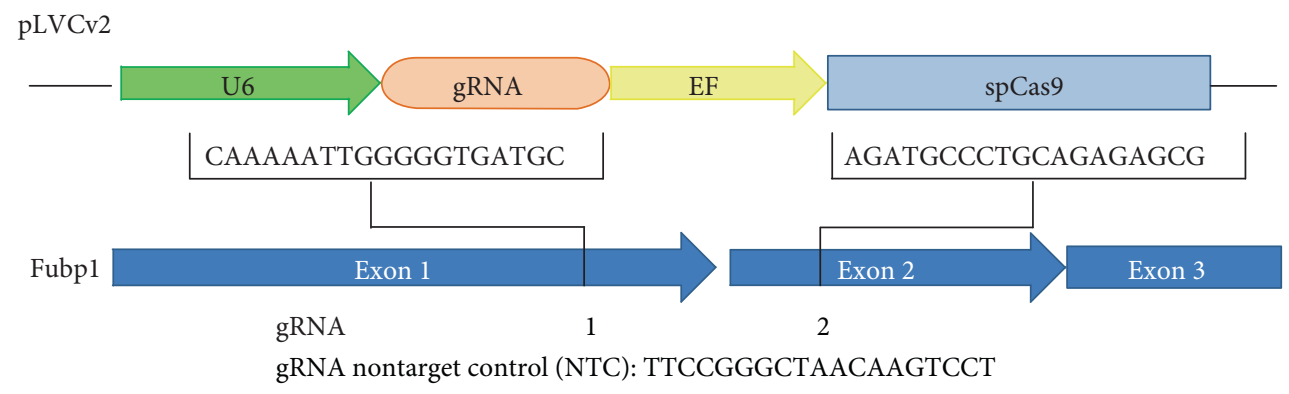

(a)

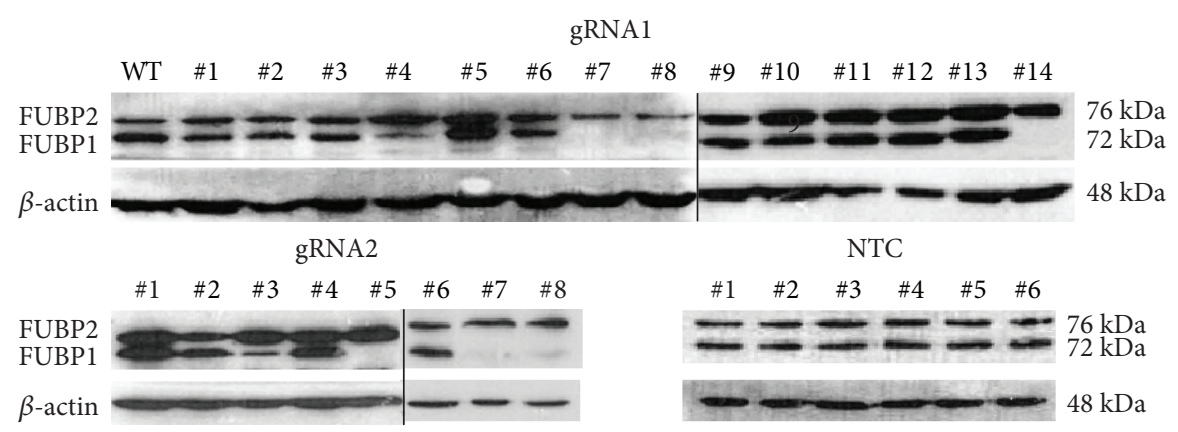

(b)
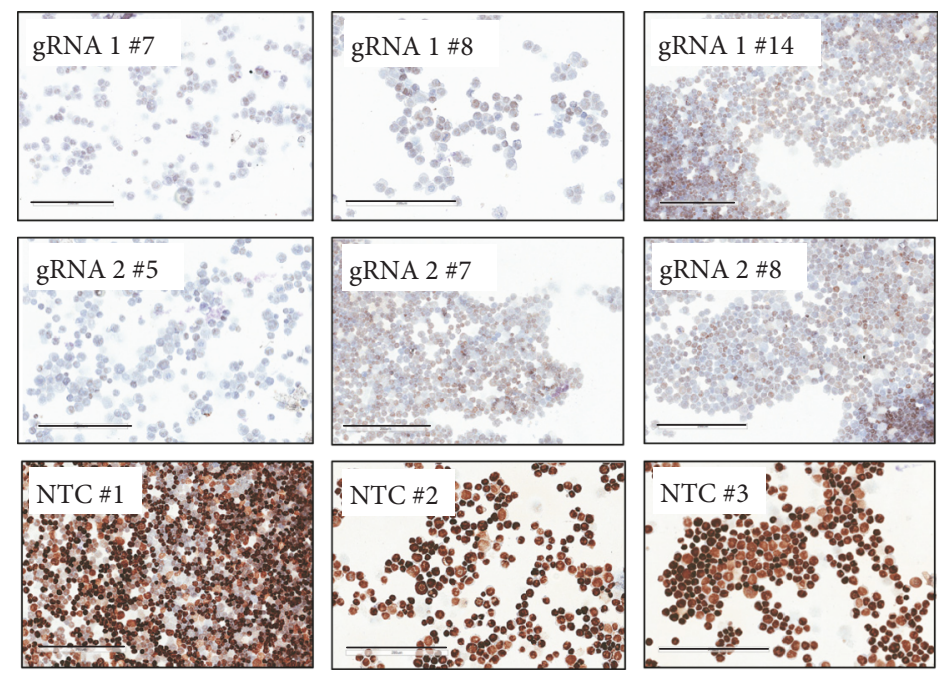

(c)

Figure 2: Generation of murine Fubp1 KO ESC clones. (a) To knockout Fubp1 in murine ESCs, two gRNA sequences were cloned into the CRISPR/Cas9 plasmid $p L C V v 2$, and one nontarget control (NTC) gRNA was cloned as a control. (b, c) FUBP1-deficient ESC clones were identified by western blot analysis (b) and by anti-FUBP1 immunohistochemistry (c) of undifferentiated cells. The weak brownish color of single Fubpl knockout cells shown in (c) is most likely due to imperfect washing-off of unbound antibody from parts of the slides with high cell density. Scale bars represent $200 \mu \mathrm{m}$.

3.3. FUBP1 Depletion Leads to a Decreased Proportion of Cells Expressing Mesoderm Marker Genes upon Aggregation into EBs. When we aggregated ESCs into EBs in LIF-deficient medium for 5 days to induce spontaneous differentiation, mRNA expression of the stem cell marker genes Oct4 and Nanog decreased as expected in both Fubpl knockout and NTC clones (Figure 3(a)). Of note, a significantly increased level of Oct4 mRNA was observed in the undifferentiated Fubp1 knockout ESC clones at day 0 of the experiment. Successful differentiation of the ESCs into cells of the three different germ layers was verified using the previously described ectoderm (Nestin and GATA4), endoderm (SOX17 and $\beta$-catenin), and mesoderm markers (Brachyury, FLK-1, SNAIL, FGFR1, and BMP4). The mRNA expression analysis of the two ectoderm marker genes Nestin and Gata4 and of the two endoderm marker genes Sox 17 and $\beta$-catenin showed no difference between FUBP1-deficient and NTC ESC clones at day 3 and day 5 of EB differentiation (Figure 3(b)). Surprisingly, when we investigated the mRNA expression of the mesoderm markers at days 3 and 5 of EB differentiation, Brachyury, Flk-1, Snail, FgfR1, and Bmp4 were significantly reduced in the Fubpl knockout cells 


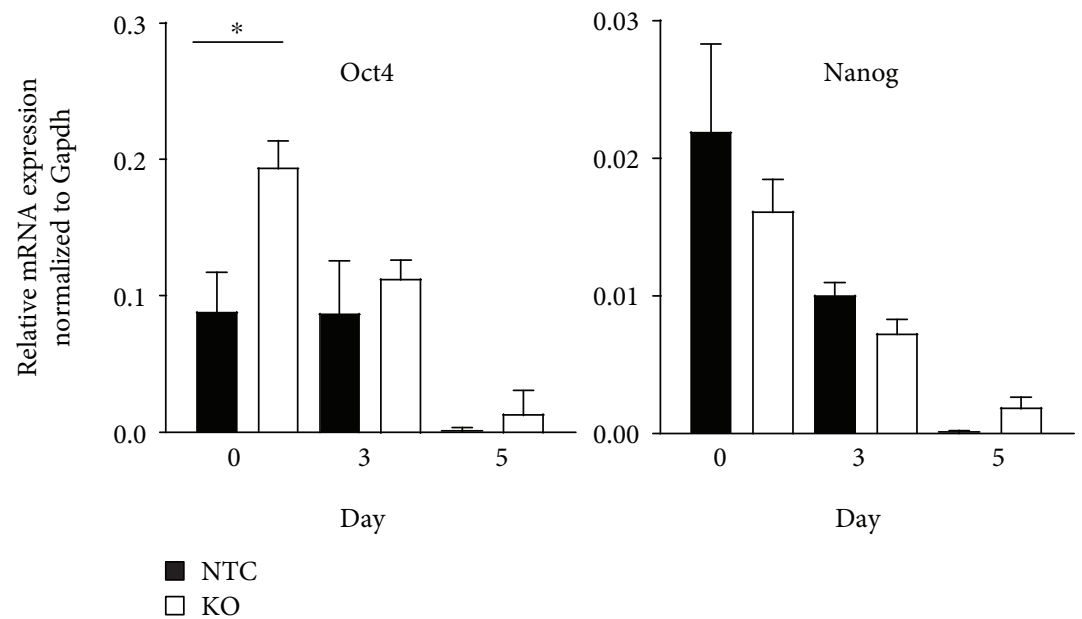

(a)
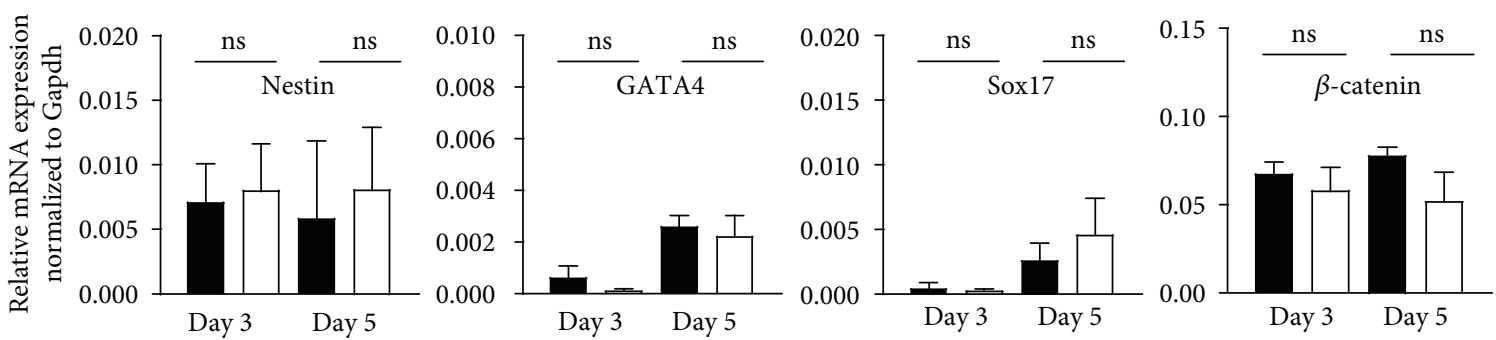

(b)
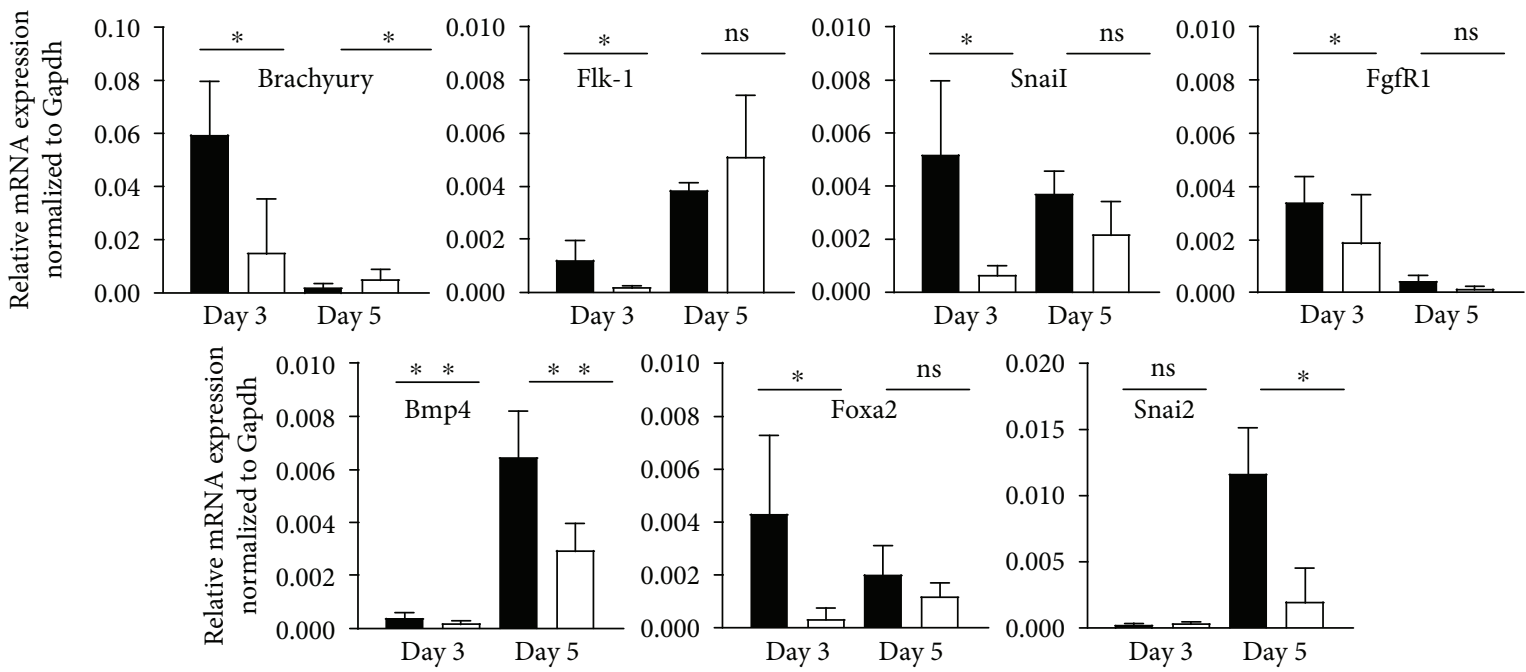

NTC

$\mathrm{KO}$

(c)

Figure 3: Spontaneous differentiation of wildtype and Fubp1 knockout ESC clones upon aggregation in EBs. (a) mRNA levels of the stem cell markers Oct4 and Nanog decreased equally in Fubp1 knockout (KO) and NTC control clones during spontaneous differentiation in EBs. (b) mRNA expression of ectoderm markers (Nestin and GATA4) slightly increased during EB formation (day 3 and day 5), but the expression was not affected by the absence of FUBP1. The same was observed for endoderm marker expression (Sox 17 and $\beta$-catenin) at days 3 and 5 . (c) The analysis of the mesoderm markers Brachyury, Flk-1, SnaiI, FGFR1, and Bmp4 revealed significantly reduced mRNA expression levels in Fubp1 $\mathrm{KO}$ clones at day 3 of ESC differentiation. In addition, the Brachyury target gene Foxa2 was significantly reduced in the Fubp1 KO compared to NTC control clones at day 3 and the Brachyury target gene Snai2 at day 5 of EB differentiation. Three independent experiments were performed, each with 4 NTC and 5 Fubp1 knockout ESC clones. The qPCR data represent the mean values \pm SD; data was normalized to Gapdh mRNA expression and calculated as relative mRNA expression level $\left({ }^{*} p<0.05 ;{ }^{* *} p<0.01\right)$. 

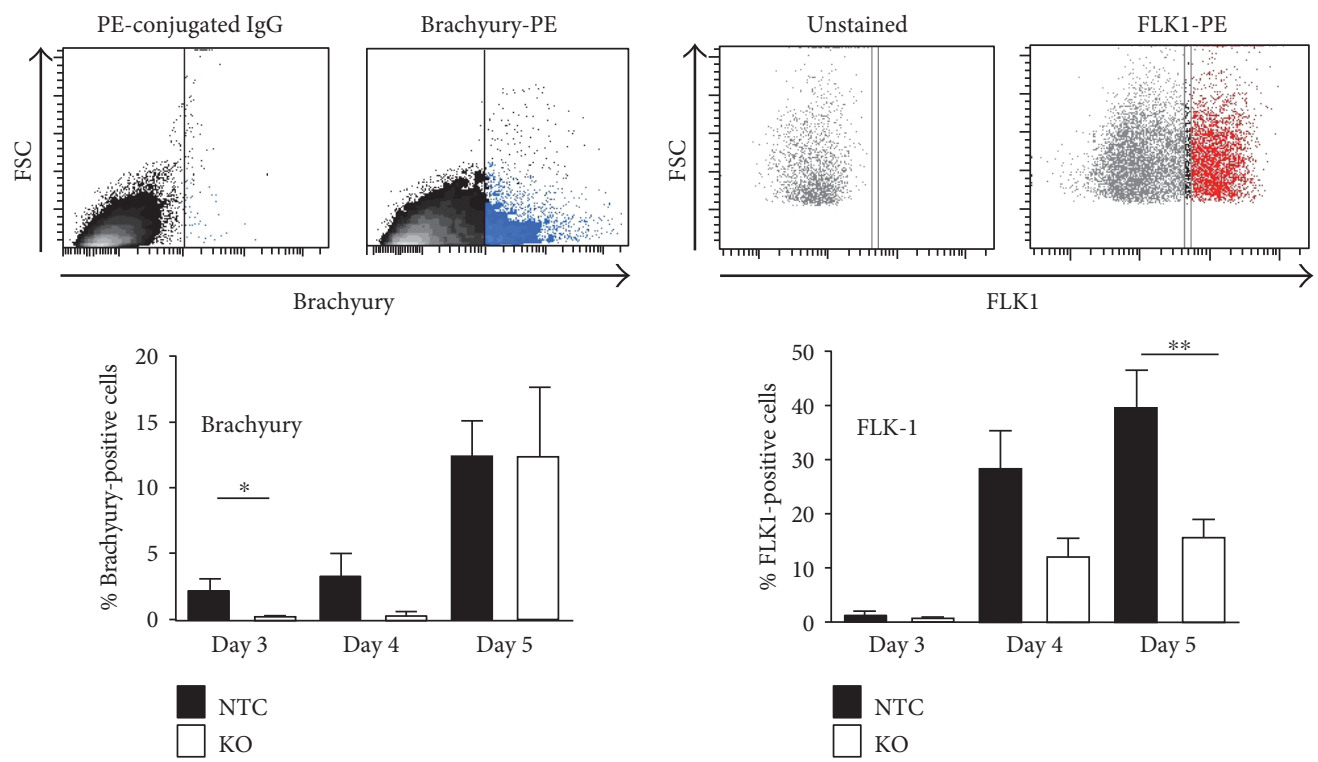

(a)

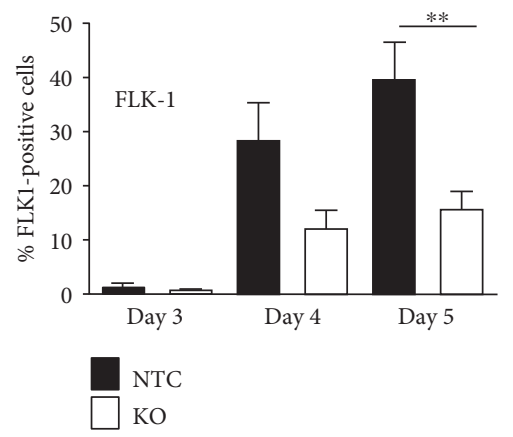

(b)
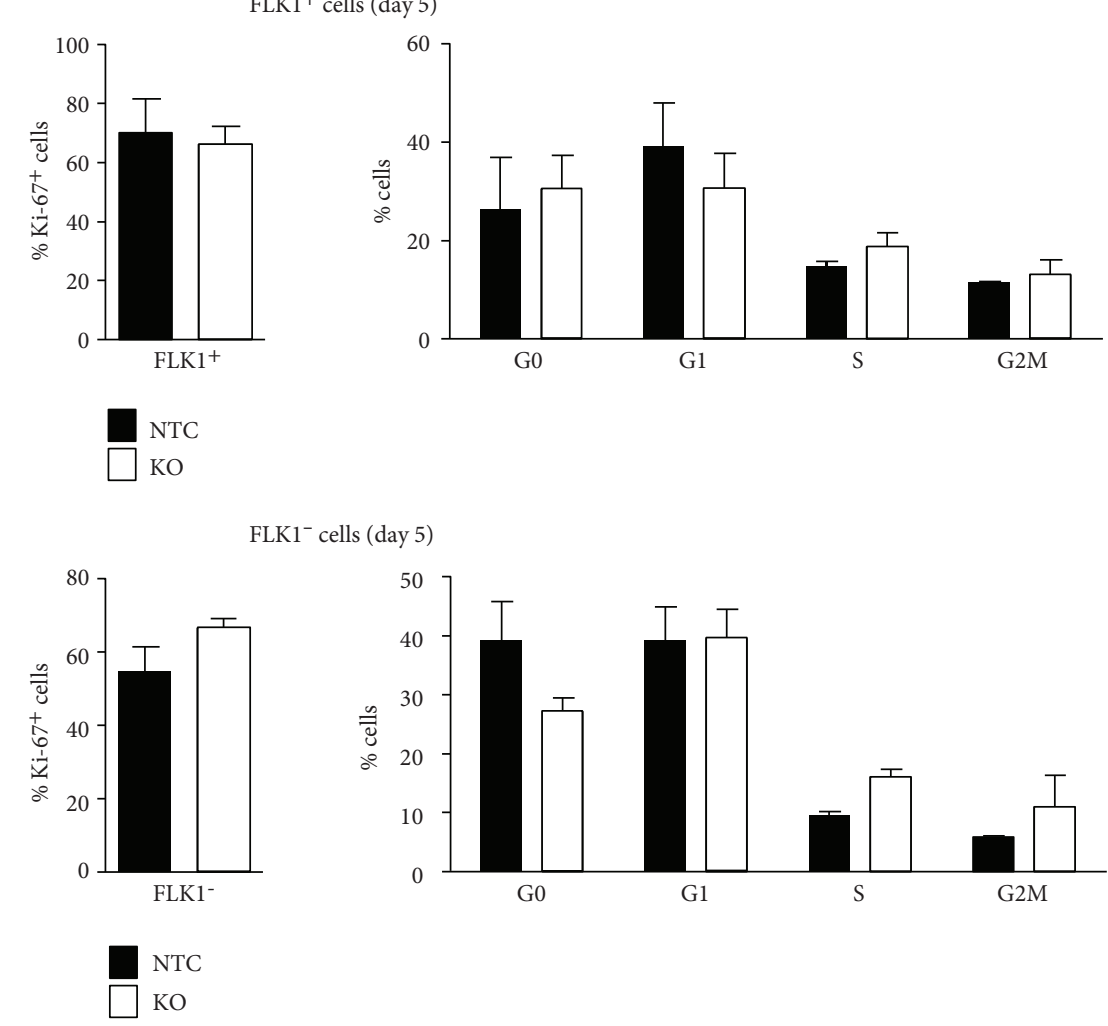

FLK1' ${ }^{-}$cells (day 5)

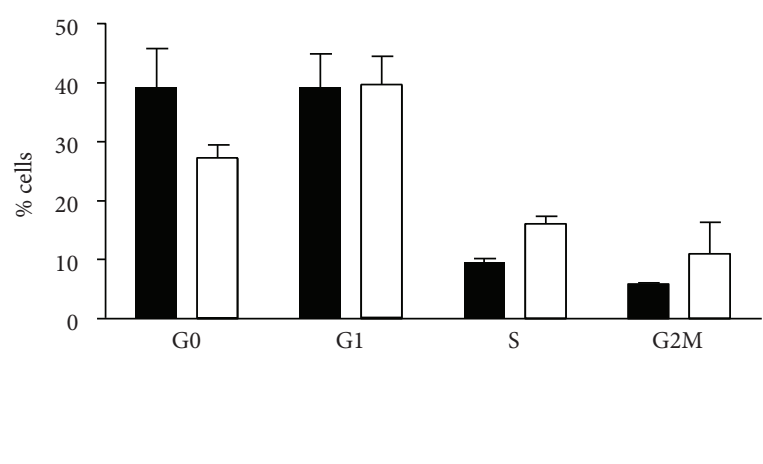

(c)

FIgURE 4: Quantification of the mesoderm marker Brachyury and FLK-1 and cell cycle analysis in cells of differentiating EBs. (a) Intracellular staining of single cells prepared from EB structures for the mesoderm marker Brachyury showed a significant reduction in the proportion of Brachyury $^{+}$Fubp1 KO cells at day 3 of EB differentiation compared to control cells derived from NTC EBs. This reduction was still visible at day 4, but not any longer at day 5 of EB differentiation, when $12 \%$ of Fubp1 KO and NTC cells derived from EBs were Brachyury ${ }^{+}$. (b) During EB differentiation, the percentage of FLK- $1^{+}$NTC cells increased from $3 \%$ (day 3) to $28 \%$ (day 4) and $39 \%$ (day 5). In comparison, the proportion of FLK $-1^{+}$Fubp1 KO cells was clearly decreased. The data represent the mean values \pm SD. In each of two independent experiments, 3 (a) or 6 (b) NTC and 3 (a) or 6 (b) Fubp1 knockout ESC clones were used $\left({ }^{*} p<0.05\right.$; $\left.{ }^{* *} p<0.01\right)$. (c) Quantification of Ki$67^{+}$cells in $\mathrm{FLK}^{+}$and $\mathrm{FLK}^{-}$cells in 3 NTC and 5 Fubp1 KO ESC clones by flow cytometry (left panels). FACS analysis was performed after 5 days of EB aggregation and differentiation in medium without LIF. Right panels: 3 NTC and 5 Fubp1 KO ESC clones were used for cell cycle analysis of $\mathrm{FLK}^{+}$and $\mathrm{FLK}^{-}$cells following 5 days of differentiation in EBs. For all experiments, data represent the mean values \pm SD. 


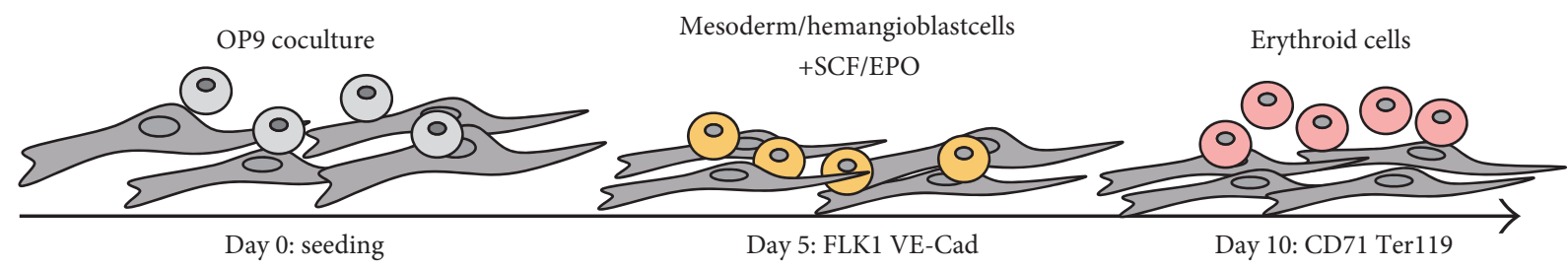

(a)
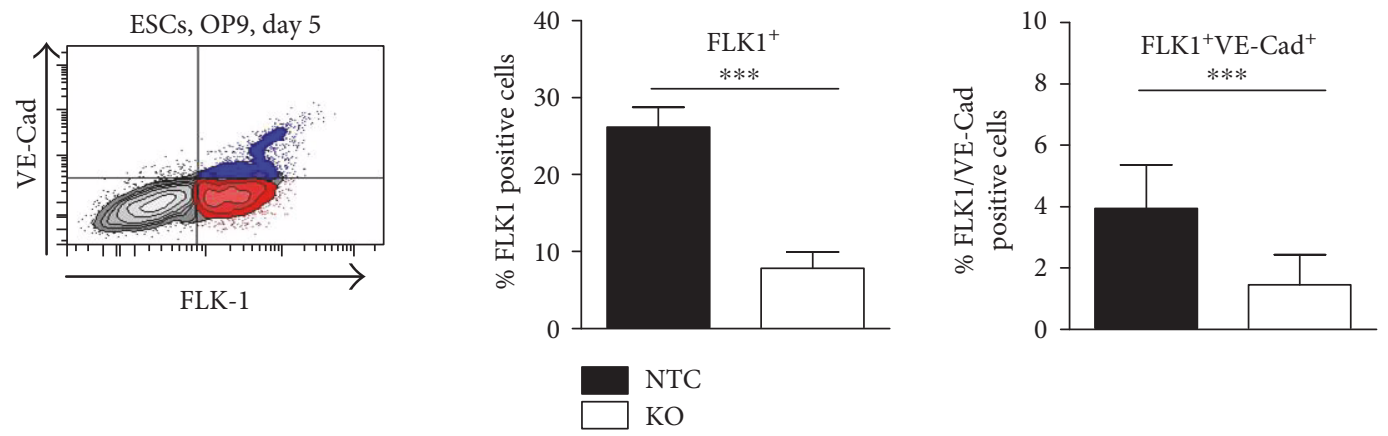

(b)

FIGURE 5: OP9 coculture assay for ESC differentiation into mesoderm/hemangioblast cells. (a) Experimental set-up for OP9 cell-induced ESC differentiation. (b) At day 5 of ESC/OP9 coculture, we analyzed the formation of mesoderm islands and hemangioblast cells and observed a significant reduction in the percentage of FUBP1-deficient $\mathrm{FLK}^{+}$and $\mathrm{FLK}-1^{+} / \mathrm{VE}-\mathrm{Cad}{ }^{+}$cells compared to NTC control cells. Three independent experiments were performed, each with 3 NTC and 5 Fubp1 knockout ESC clones. The data represent the mean values \pm SD $\left({ }^{* * *} p<0.001\right)$.

compared to the NTC controls (Figure 3(c)). In line with the decreased Brachyury expression in the Fubp1 knockout cells, mRNA levels of the Brachyury target genes Foxa2 [34] and Snai2 [35] were diminished in the FUBP1-deficient differentiating ESCs compared to cells with wildtype FUBP1 expression (Figure 3(c)).

The mRNA expression data were supported by flow cytometry experiments. Figure 4(a) demonstrates that the amount of intracellularly stained Brachyury ${ }^{+}$cells at days 3 and 4 of EB differentiation was reduced in Fubpl knockout cells compared to NTC clones, while at day 5, the amount of Brachyury ${ }^{+}$cells was comparable. The quantification of FLK $-1^{+}$cells by flow cytometry showed a relatively small number of FLK-1-expressing cells at day 3 of EB differentiation (Figure 4(b)). However, at day 4, 30\% of all differentiating NTC cells became FLK1-positive, while only $15 \%$ of the Fubp1 knockout cells had started to express FLK1. This difference became even more obvious at day 5 of differentiation (18\% FLK $^{+}$Fubp1 knockout cells versus $40 \%$ FLK $^{+}$NTC cells), supporting the conclusion that FUBP deficiency in ESCs leads to delayed mesoderm differentiation. Additionally, Fubp1 mRNA levels were slightly increased in a FACSsorted FLK $-1^{+}$cell population at day 4 of EB differentiation compared to $\mathrm{FLK}^{-}$cells (Figure S4).

Our previous work had identified a pro-proliferative function of FUBP1 in HSCs [6] and HCC cells [4]. To investigate a potential cell type-specific proliferation defect in differentiating Fubp1 knockout ESCs that would explain the delayed production of mesoderm cells in the absence of FUBP1, we analyzed the proportion of cycling (Ki-67 positive) $\mathrm{FLK}^{+}$and $\mathrm{FLK} 1^{-}$cells by flow cytometry. In addition, we measured the cell cycle phase distribution of the cells
(Figure 4(c)). Overall, we could not detect significant differences in the proliferative behavior of NTC control and Fubpl knockout ESC clones, neither in the $\mathrm{FLK}^{+}$nor in the $\mathrm{FLK} 1^{-}$ subpopulation of differentiating ESCs.

3.4. The Absence of FUBP1 Leads to Diminished Erythroid Differentiation. Homozygous Fubpl genetrap mouse embryos lacking FUBP1 activity die in utero with a severe anemic phenotype that is explained by dysfunctional HSC self-renewal [6]. In addition, we had noticed an accumulation of erythroid progenitors during fetal erythropoiesis. The delayed generation of mesoderm cells in the absence of FUBP1 could affect the generation of the hematopoietic lineage. To address this possibility, we used the established coculture assay of ESCs with OP9 cells, which do not produce functional macrophage colony-stimulating factor (M-CSF; $[27,28])$, to induce hematopoietic differentiation of ESCs and to analyze the consequences of FUBP1 deficiency for erythropoiesis (see Figure 5(a)). We observed a reduced number of $\mathrm{FLK}^{+}$mesoderm cells and FLK-1 ${ }^{+} / \mathrm{VE}-\mathrm{Cad}^{+}$ hemangioblasts in the Fubpl knockout cultures compared to the differentiating wildtype ESCs at day 5 of ESC/OP-9 coculture (Figure 5(b)). However, the analysis of CD $45^{+}$cells at day 12 of OP-9 coculture differentiation (with SCF, but without EPO) resulted in no obvious difference between Fubp1 knockout and NTC clones (Figure S5).

Upon specific differentiation into the erythroid lineage by supplementation of the medium with the cytokines stem cell factor (SCF) and erythropoietin (EPO) at day 5 of ESC/OP9 coculture [28], we could generate a high percentage of different maturation stages of the erythroid lineage. Of note, the amount of $\mathrm{CD}^{-} 1^{-}$Ter119- ESCs that were not differentiated 

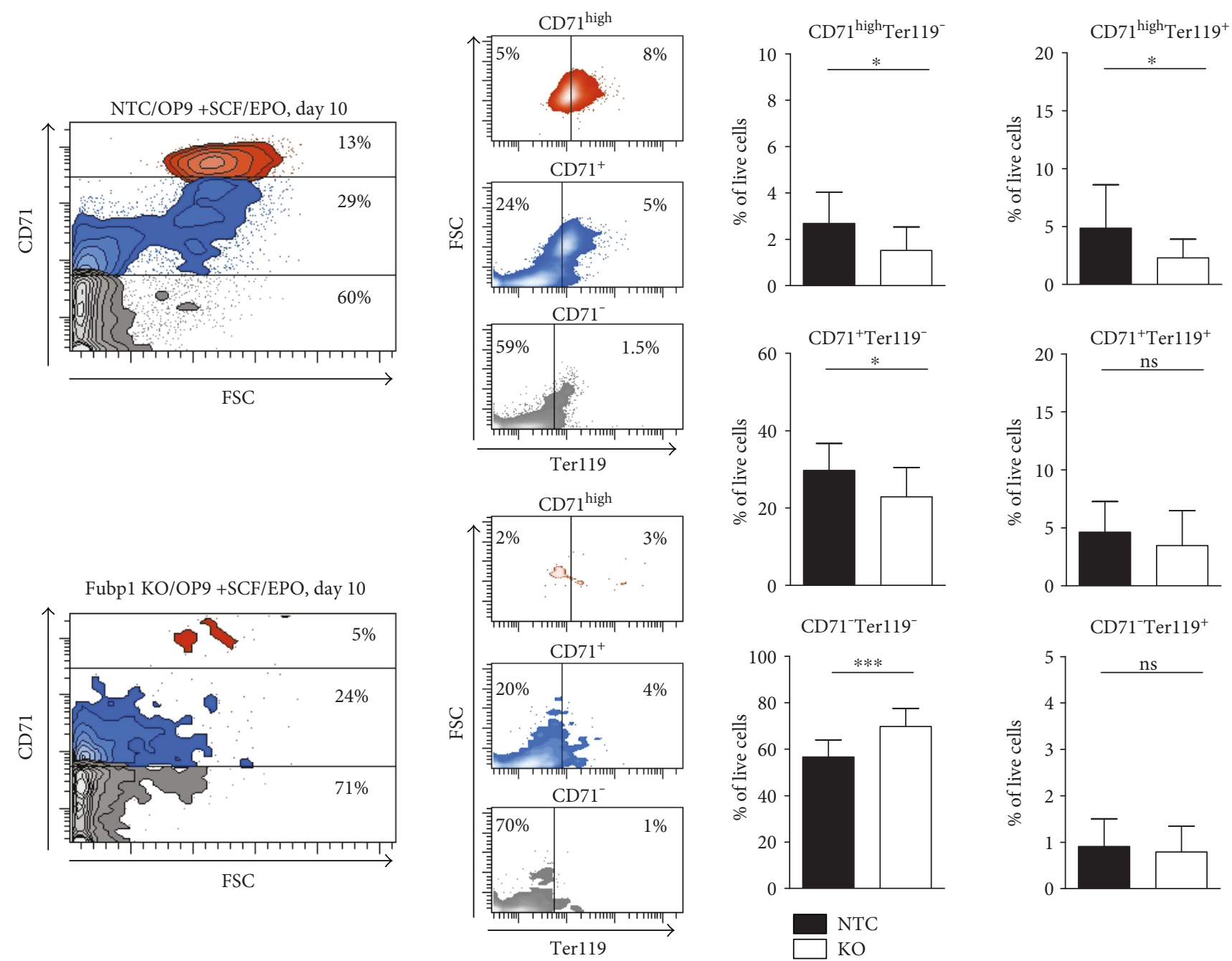

FIGURE 6: ESC/OP9 coculture in the presence of SCF and EPO for erythroid differentiation. The erythroid differentiation of Fubp1 KO ESCs was significantly reduced compared to NTC control ESCs, while the amount of CD71 $/$ Ter119- ESCs that were not differentiated into the erythroid lineage was increased in the Fubp1 KO clones (70\% KO versus 59\% NTC). Maturation of erythroid cells started with the

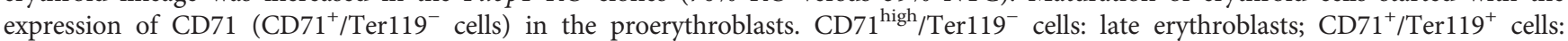
reticulocytes; CD71-/Ter119+: mature red blood cells. Three independent experiments were performed, each with 3 NTC and 6 Fubp 1 knockout ESC clones. The data represent the mean values \pm SD $\left({ }^{*} p<0.05 ;{ }^{* * *} p<0.001\right)$.

into the erythroid lineage was increased in the Fubpl KO compared to the NTC control clones (69.7\% versus $56.6 \%$ ), reflecting a reduced erythroid differentiation of Fubp1 KO ESCs in the absence of FUBP1 (Figure 6). For the detailed analysis of erythroid marker expression by flow cytometry, we took the morphological changes of the ESCs during erythroid differentiation into account and discriminated first the three $\mathrm{CD}_{71}{ }^{-}, \mathrm{CD}_{7}{ }^{+}$, and $\mathrm{CD} 71^{\text {high }}$ cell populations, before analyzing the Ter119 expression separately for each of these three CD71 subpopulations (Figure 6). Erythroid differentiation started with the expression of CD71 $(22.8 \%$ $\mathrm{CD}_{71}{ }^{+} /$Ter1 $19^{-} \mathrm{KO}$ versus $27.9 \% \mathrm{CD}^{-} 1^{+} / \mathrm{Ter} 119^{-} \mathrm{NTC}$ cells) in the proerythroblasts. The maturation into early erythroblast cells was indicated by CD71 ${ }^{\text {high }} /$ Ter $119^{-}$expression (1.4\% KO versus $2.7 \%$ NTC), and late erythroblasts cells expressed CD71 $1^{\text {high }} /$ Ter $119^{+}$(2.3\% KO versus $4.9 \%$ NTC). The cells matured further into $\mathrm{CD} 71^{+} / \mathrm{Ter} 119^{+}$reticulocytes
(3.4\% KO versus $4.6 \%$ NTC) and finally into mature CD71 $1^{-}$Ter $119^{+}$red blood cells $(0.8 \% \mathrm{KO}$ versus $0.9 \%$ NTC).

\section{Discussion}

FUBP1 controls a complex transcriptional network in cells by binding to AT-rich FUSE DNA sequences and influencing the transcription of numerous target genes like $c-m y c, p 21$, USP29, and others [36]. Melting of the FUSE DNA sequences, that is, the occurrence of single-stranded DNA structures and their recognition by FUBP1, serves as an additional control level to regulate transcription. Most of the FUBP1 literature centers on its role in tumorigenesis, and only recently, an essential physiological role has been described for the protein in HSC self-renewal [6, 7]. In both biological systems, HSCs and FUBP1 (over-) expressing tumor cells, the FUBP1 transcription network provides pro- 

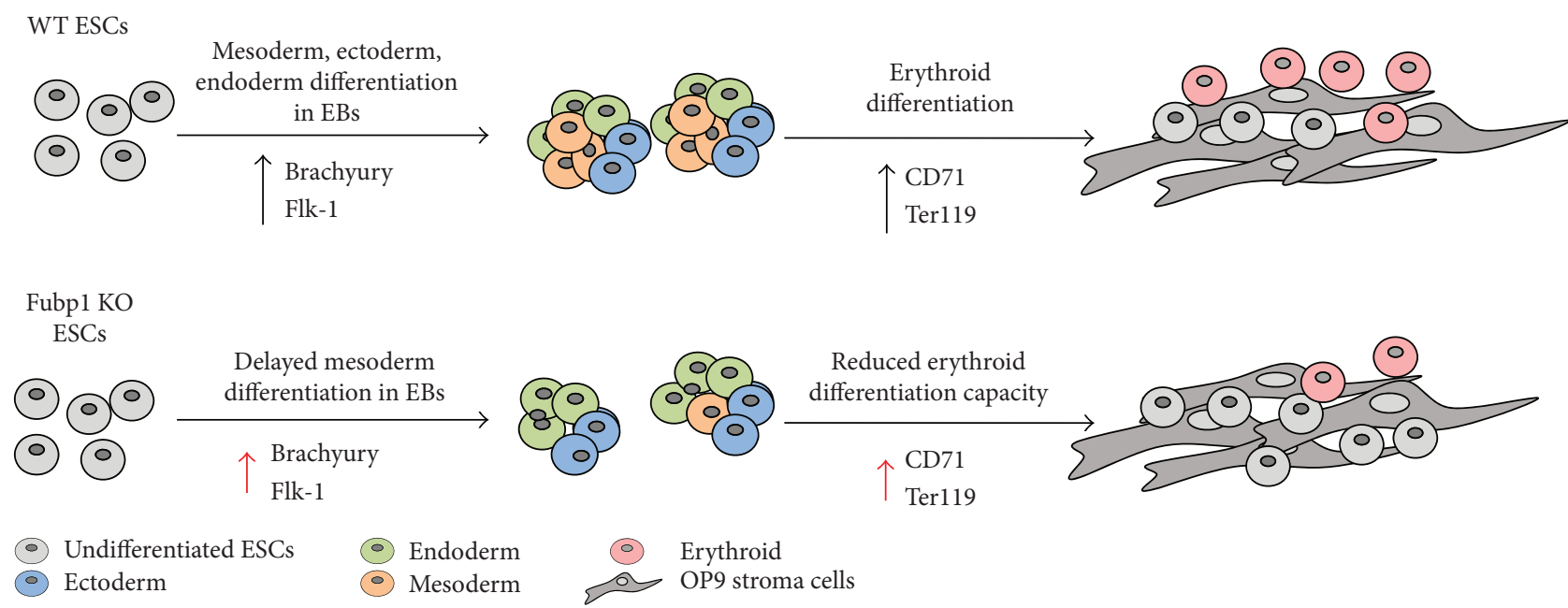

FIgURE 7: The absence of FUBP1 leads to a delayed mesoderm marker expression during EB formation and a decreased production of mesoderm cells, resulting in a significantly reduced differentiation into erythroid cells.

proliferative and antiapoptotic activity $[4,6]$. Based on its prominent stem cell function in HSCs, we now investigated a potential role for FUBP1 in ESCs. We noticed a rather heterogeneous FUBP1 expression level in undifferentiated single ESCs, and during differentiation into the three germ layers in EBs, the overall expression of FUBP1 decreased, probably because a proportion of cells stopped producing FUBP1 protein at all. Surprisingly, removal of FUBP1 expression in murine ESCs using CRISPR/Cas9 technology did not interfere with ESC maintenance and pluripotency (as judged by SSEA1/SSEA4 marker expression). The cell cycle distribution, spontaneous cell death rate, and self-renewal of FUBP1-deficient ESCs did not change compared to NTC control clones. However, upon aggregation of Fubp1 KO ESCs into EBs and spontaneous differentiation into cells of the three germ layers [19], we observed a significant reduction of mesoderm cell differentiation as shown by the analysis of a variety of mesoderm markers, such as Brachyury and Flk-1 [35, 37, 38]. In contrast, ectoderm and endoderm marker expression was not altered in the absence of FUBP1. As a consequence of the reduced Brachyury levels, the Brachyury target genes Snai2 [34] and Foxa2 [35] were significantly reduced in the absence of FUBP1. Although we did not include every germ lineage marker available in our analysis, our data suggest that the accurate enhancement of mesoderm marker expression cannot be implemented at the beginning of EB differentiation in FUBP1-deficient ESCs. This implicates a delayed and insufficient mesoderm cell differentiation, while, at the same time, lack of FUBP1 does not affect proper ESC differentiation into the ectoderm and endoderm lineages in EBs (see Figure 7). Of note, the hematopoietic cell lineages are derived from mesoderm germ layer, and it is well possible that the impaired self-renewal seen in FUBP1-deficient HSCs [6] is connected to our observed defect in mesoderm differentiation. An alternative explanation could be a cell type-specific proliferation defect in FUBP1-deficient differentiating ESCs that would result in an apparent delay of the production of this particular cell type. However, our cell cycle analysis of FUBP1-expressing and FUBP1-deficient $\mathrm{FLK}^{+}$and $\mathrm{FLK}^{-}$cells after 5 days of spontaneous differentiation in EBs did not support such FUBP1-dependent differences in the proliferative capacity of particular cell populations.

Mesoderm cells can be further differentiated into hematopoietic cell lineages $[39,40]$. In addition to the diminished HSC self-renewal, we had observed an increase in immature erythroid progenitors in Fubp1 mutant E15.5 embryos, suggesting a differentiation defect in the red blood cell line [6]. The delayed mesoderm differentiation in Fubp1 knockout ESCs prompted us to differentiate the ESC culture further towards erythropoiesis. In our OP9 coculture system with EPO/SCF supply, we found a higher proportion of less mature FUBP1-deficient cells, resulting in a diminished proportion of further differentiated CD $71^{+}$Ter $119^{+}$erythroid cells. Our data support a physiological role of FUBP1 for the differentiation of immature progenitors into functional erythrocytes that extends its HSCs-specific function in hematopoiesis (see Figure 7). Further investigations will aim to identify the relevant target genes within the transcriptional FUBP1 network that are important for timely mesoderm differentiation and proper erythrocyte maturation.

\section{Conclusion}

In the present study, we examined the role of the transcriptional regulator FUBP1 during maintenance and differentiation of ESCs. For this purpose, we generated FUBP1-deficient ESC clones using CRISPR/Cas9 technology. Surprisingly and in contrast to HSCs, FUBP1 seems to be dispensable for ESC self-renewal, and the absence of FUBP1 does not result in reduced proliferation or increased embryonic stem cell death. However, when we aggregated ESCs to EBs for spontaneous differentiation into cells of all three germ layers, we noted a profound delay in mesoderm differentiation from Fubp1 knockout ESCs. Expression levels of several mesoderm markers, including Brachyury, were significantly reduced during differentiation. Cells of the hematopoietic lineage are derived from mesoderm, and in line with the observed 
mesoderm differentiation defect, we noticed an impaired differentiation capacity of FUBP1-deficient ESCs into the erythroid lineage. Our results substantiate the notion that the proper regulation of the cell type-specific transcriptional network that is controlled by FUBP1 is crucial not only for HSC self-renewal but also for the appropriate differentiation during erythropoiesis.

\author{
Abbreviations \\ CRISPR: Clustered regularly interspaced short palindromic \\ repeats \\ EtOH: Ethanol \\ EB: $\quad$ Embryoid body \\ EPO: Eryhropoietin \\ ESC: $\quad$ Embryonic stem cell \\ FUBP1: FUSE binding protein 1 \\ FUSE: Far upstream element \\ gRNA: Guide RNA \\ HSC: Hematopoietic stem cell \\ IHC: Immunohistochemistry \\ KO: $\quad$ Knockout \\ LIF: Leukemia inhibitory factor \\ M-CSF: Macrophage colony-stimulating factor \\ NTC: Nontarget control \\ qPCR: Quantitative polymerase chain reaction \\ SCF: $\quad$ Stem cell factor.
}

\section{Disclosure}

The authors are solely responsible for the contents and writing of this paper.

\section{Conflicts of Interest}

The authors declare no conflict of interests.

\section{Authors' Contributions}

Josephine Wesely and Marlene Steiner contributed to the acquisition, analysis and interpretation of data, and drafting of the manuscript. Frank Schnütgen and Manuel Kaulich contributed to the material support, experimental design, analysis, and interpretation of data. Michael A. Rieger contributed to the experimental design, analysis and interpretation of data, and critical revision of the manuscript. Martin Zörnig contributed to the experimental design, analysis and interpretation of data, and drafting of the manuscript. All authors have approved the final version of the manuscript.

\section{Acknowledgments}

The authors are thankful to Susanne Bösser and Claudia Jordan for excellent technical assistance, to Frederic B. Thalheimer and Stefan Stein for help and advice with the flow cytometry experiments and the OP9 coculture experiments, and to Klaus Lehmen for his help in preparing the figures. This work was supported by the German Research Foundation (no. ZO 110/7-1), the Wilhelm Sander-Stiftung (no. 2015.039.1), the German Cancer Consortium (MZ), the Stiftung Polytechnische Gesellschaft Frankfurt am Main (M.S.), the LOEWE Center for Cell and Gene Therapy Frankfurt (M.A.R, MZ), and the Hessisches Ministerium für Wissenschaft und Kunst (III L 4- 518/17.004 [2014]).

\section{References}

[1] M. I. Avigan, B. Strober, and D. Levens, "A far upstream element stimulates c-myc expression in undifferentiated leukemia cells," The Journal of Biological Chemistry, vol. 265, no. 30, pp. 18538-18545, 1990.

[2] M. Y. Jia and Y. J. Wang, "Far upstream element-binding protein 1(FUBP1) expression differs between human colorectal cancer and non-cancerous tissue," Neoplasma, vol. 61, no. 5, pp. 533-540, 2014.

[3] M. Malz, A. Weber, S. Singer et al., "Overexpression of far upstream element binding proteins: a mechanism regulating proliferation and migration in liver cancer cells," Hepatology, vol. 50, no. 4, pp. 1130-1139, 2009.

[4] U. Rabenhorst, R. Beinoraviciute-Kellner, M. L. Brezniceanu et al., "Overexpression of the far upstream element binding protein 1 in hepatocellular carcinoma is required for tumor growth," Hepatology, vol. 50, no. 4, pp. 1121-1129, 2009.

[5] A. Weber, I. Kristiansen, M. Johannsen et al., "The FUSE binding proteins FBP1 and FBP3 are potential c-myc regulators in renal, but not in prostate and bladder cancer," BMC Cancer, vol. 8 , no. 1, p. $369,2008$.

[6] U. Rabenhorst, F. B. Thalheimer, K. Gerlach et al., "Singlestranded DNA-binding transcriptional regulator FUBP1 is essential for fetal and adult hematopoietic stem cell selfrenewal," Cell Reports, vol. 11, no. 12, pp. 1847-1855, 2015.

[7] W. Zhou, Y. J. Chung, E. R. Parrilla Castellar et al., "Far upstream element binding protein plays a crucial role in embryonic development, hematopoiesis, and stabilizing Myc expression levels," The American Journal of Pathology, vol. 186, no. 3, pp. 701-715, 2016.

[8] J. Wray, T. Kalkan, and A. G. Smith, "The ground state of pluripotency," Biochemical Society Transactions, vol. 38, no. 4, pp. 1027-1032, 2010.

[9] G. Martello and A. Smith, "The nature of embryonic stem cells," Annual Review of Cell and Developmental Biology, vol. 30, no. 1, pp. 647-675, 2014.

[10] A. G. Smith and M. L. Hooper, "Buffalo rat liver cells produce a diffusible activity which inhibits the differentiation of murine embryonal carcinoma and embryonic stem cells," Developmental Biology, vol. 121, no. 1, pp. 1-9, 1987.

[11] Y. Avior, I. Sagi, and N. Benvenisty, "Pluripotent stem cells in disease modelling and drug discovery," Nature Reviews. Molecular Cell Biology, vol. 17, no. 3, pp. 170-182, 2016.

[12] K. Li, Y. Kong, M. Zhang, F. Xie, P. Liu, and S. Xu, "Differentiation of pluripotent stem cells for regenerative medicine," Biochemical and Biophysical Research Communications, vol. 471, no. 1, pp. 1-4, 2016.

[13] V. Picanco-Castro, L. F. Moreira, S. Kashima, and D. T. Covas, "Can pluripotent stem cells be used in cell-based therapy?" Cellular Reprogramming, vol. 16, no. 2, pp. 98-107, 2014.

[14] E. Kingham and R. O. Oreffo, "Embryonic and induced pluripotent stem cells: understanding, creating, and exploiting the nano-niche for regenerative medicine," ACS Nano, vol. 7, no. 3, pp. 1867-1881, 2013. 
[15] I. Klimanskaya, N. Rosenthal, and R. Lanza, "Derive and conquer: sourcing and differentiating stem cells for therapeutic applications," Nature Reviews. Drug Discovery, vol. 7, no. 2, pp. 131-142, 2008.

[16] H. Wang, X. Luo, and J. Leighton, "Extracellular matrix and integrins in embryonic stem cell differentiation," Biochemistry Insights, vol. 8, Supplement 2, pp. 15-21, 2015.

[17] E. Garcia-Alegria, S. Menegatti, K. Batta, S. Cuvertino, M. Florkowska, and V. Kouskoff, "Emerging concepts for the in vitro derivation of murine haematopoietic stem and progenitor cells," FEBS Letters, vol. 590, no. 22, pp. 4116-4125, 2016.

[18] L. Wahlster and G. Q. Daley, "Progress towards generation of human haematopoietic stem cells," Nature Cell Biology, vol. 18, no. 11, pp. 1111-1117, 2016.

[19] M. Koike, S. Sakaki, Y. Amano, and H. Kurosawa, "Characterization of embryoid bodies of mouse embryonic stem cells formed under various culture conditions and estimation of differentiation status of such bodies," Journal of Bioscience and Bioengineering, vol. 104, no. 4, pp. 294-299, 2007.

[20] H. Kurosawa, "Methods for inducing embryoid body formation: in vitro differentiation system of embryonic stem cells," Journal of Bioscience and Bioengineering, vol. 103, no. 5, pp. 389-398, 2007.

[21] Y. C. Poh, J. Chen, Y. Hong, H. Yi, S. Zhang, J. Chen et al., "Generation of organized germ layers from a single mouse embryonic stem cell," Nature Communications, vol. 5, article 4000, p. 1, 2014.

[22] A. C. Komor, A. H. Badran, and D. R. Liu, "CRISPR-based technologies for the manipulation of eukaryotic genomes," Cell, vol. 168, no. 1-2, pp. 20-36, 2016.

[23] L. A. Boyer, T. I. Lee, M. F. Cole et al., "Core transcriptional regulatory circuitry in human embryonic stem cells," Cell, vol. 122, no. 6, pp. 947-956, 2005.

[24] I. Chambers and S. R. Tomlinson, "The transcriptional foundation of pluripotency," Development, vol. 136, no. 14, pp. 2311-2322, 2009.

[25] K. Hawkins, S. Joy, and T. McKay, "Cell signalling pathways underlying induced pluripotent stem cell reprogramming," World Journal of Stem Cells, vol. 6, no. 5, pp. 620-628, 2014.

[26] G. Huang, S. Ye, X. Zhou, D. Liu, and Q. L. Ying, "Molecular basis of embryonic stem cell self-renewal: from signaling pathways to pluripotency network," Cellular and Molecular Life Sciences, vol. 72, no. 9, pp. 1741-1757, 2015.

[27] T. Nakano, H. Kodama, and T. Honjo, "Generation of lymphohematopoietic cells from embryonic stem cells in culture," Science, vol. 265, no. 5175, pp. 1098-1101, 1994.

[28] J. Zheng, K. Kitajima, E. Sakai et al., "Differential effects of GATA-1 on proliferation and differentiation of erythroid lineage cells," Blood, vol. 107, no. 2, pp. 520-527, 2006.

[29] M. Hooper, K. Hardy, A. Handyside, S. Hunter, and M. Monk, "HPRT-deficient (Lesch-Nyhan) mouse embryos derived from germline colonization by cultured cells," Nature, vol. 326, no. 6110, pp. 292-295, 1987.

[30] N. E. Sanjana, O. Shalem, and F. Zhang, "Improved vectors and genome-wide libraries for CRISPR screening," Nature Methods, vol. 11, no. 8, pp. 783-784, 2014.

[31] J. H. Schefe, K. E. Lehmann, I. R. Buschmann, T. Unger, and H. Funke-Kaiser, "Quantitative real-time RT-PCR data analysis: current concepts and the novel "gene expression's CT difference" formula," Journal of Molecular Medicine (Berlin), vol. 84, no. 11, pp. 901-910, 2006.
[32] T. Brambrink, R. Foreman, G. G. Welstead et al., "Sequential expression of pluripotency markers during direct reprogramming of mouse somatic cells," Cell Stem Cell, vol. 2, no. 2, pp. 151-159, 2008.

[33] W. Zhao, X. Ji, F. Zhang, L. Li, and L. Ma, "Embryonic stem cell markers," Molecules, vol. 17, no. 6, pp. 6196-6236, 2012.

[34] M. Lolas, P. D. Valenzuela, R. Tjian, and Z. Liu, "Charting Brachyury-mediated developmental pathways during early mouse embryogenesis," Proceedings of the National Academy of Sciences of the United States of America, vol. 111, no. 12, pp. 4478-4483, 2014.

[35] A. L. Evans, T. Faial, M. J. Gilchrist et al., "Genomic targets of Brachyury (T) in differentiating mouse embryonic stem cells," PloS One, vol. 7, no. 3, article e33346, 2012.

[36] J. Zhang and Q. M. Chen, "Far upstream element binding protein 1: a commander of transcription, translation and beyond," Oncogene, vol. 32, no. 24, pp. 2907-2916, 2013.

[37] T. Faial, A. S. Bernardo, S. Mendjan et al., "Brachyury and SMAD signalling collaboratively orchestrate distinct mesoderm and endoderm gene regulatory networks in differentiating human embryonic stem cells," Development, vol. 142, no. 12, pp. 2121-2135, 2015.

[38] S. I. Nishikawa, S. Nishikawa, M. Hirashima, N. Matsuyoshi, and H. Kodama, "Progressive lineage analysis by cell sorting and culture identifies FLK1+VE-cadherin + cells at a diverging point of endothelial and hemopoietic lineages," Development, vol. 125, no. 9, pp. 1747-1757, 1998.

[39] T. Inoue-Yokoo, K. Tani, and D. Sugiyama, "Mesodermal and hematopoietic differentiation from ES and iPS cells," Stem Cell Reviews, vol. 9, no. 4, pp. 422-434, 2013.

[40] H. K. Mikkola and S. H. Orkin, "The journey of developing hematopoietic stem cells," Development, vol. 133, no. 19, pp. 3733-3744, 2006. 

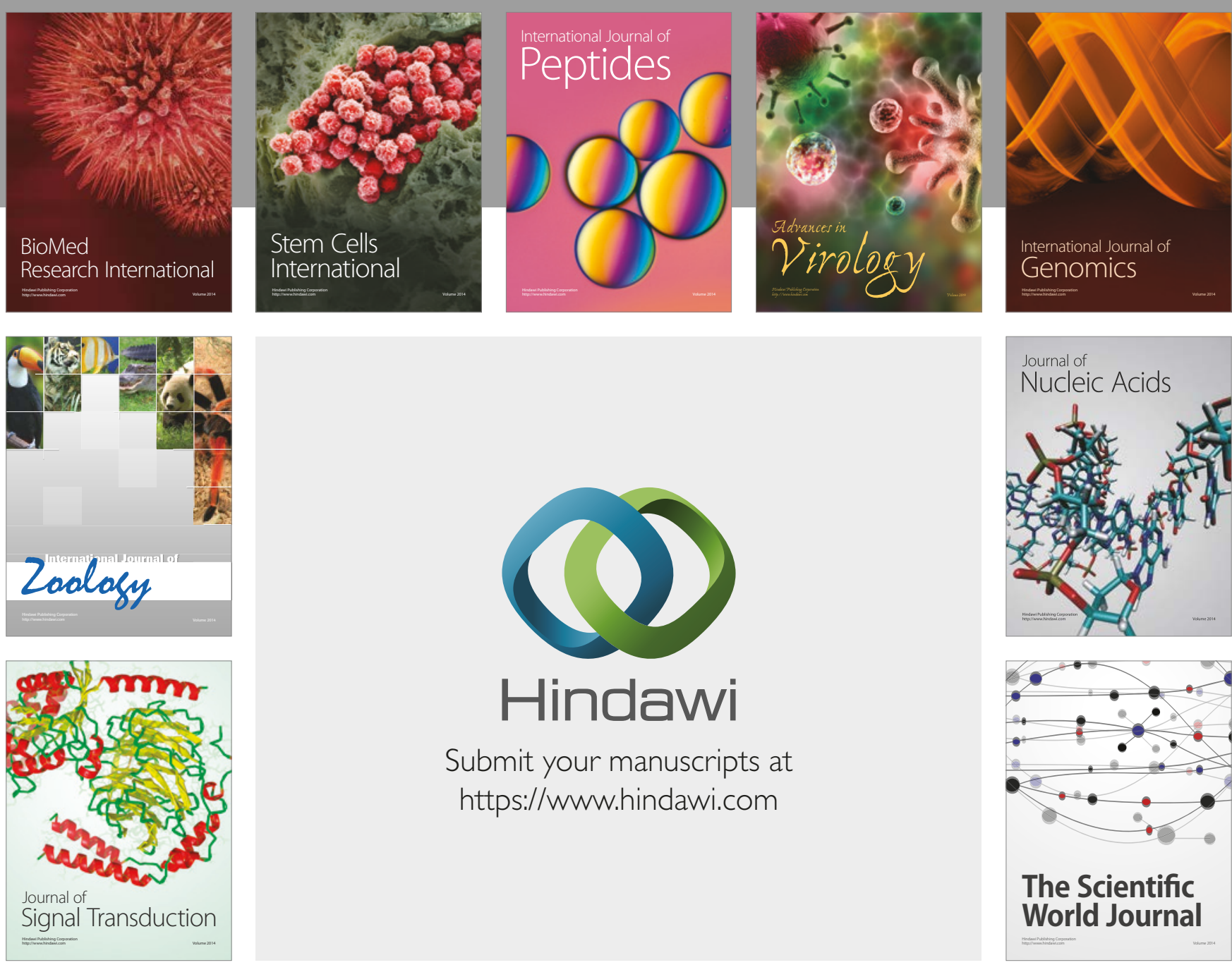

Submit your manuscripts at

https://www.hindawi.com
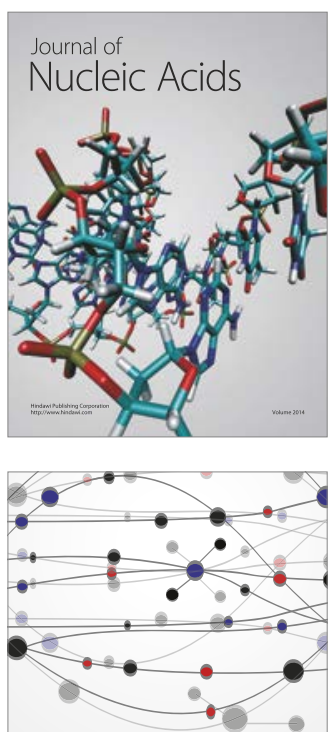

The Scientific World Journal

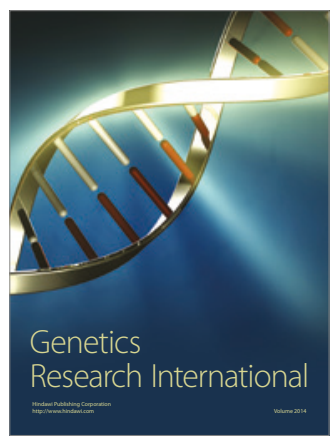

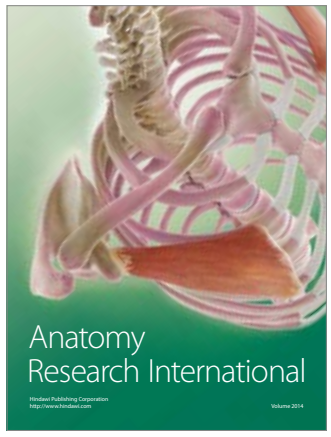

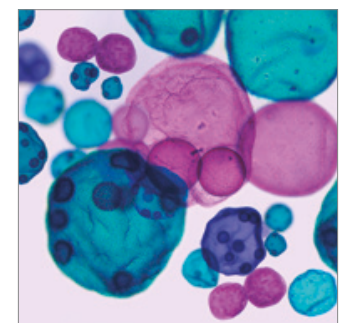

International Journal of Microbiology
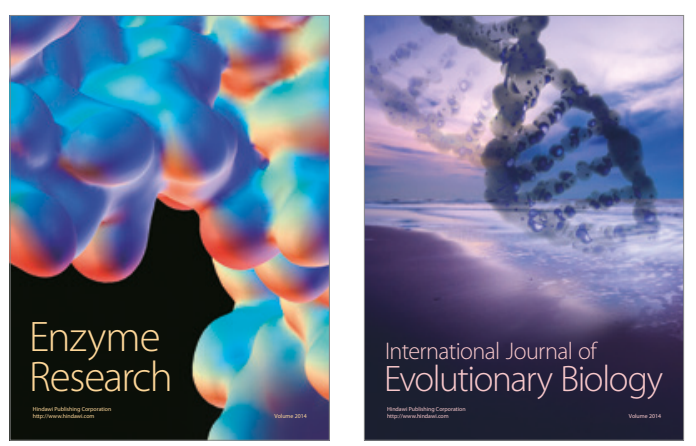
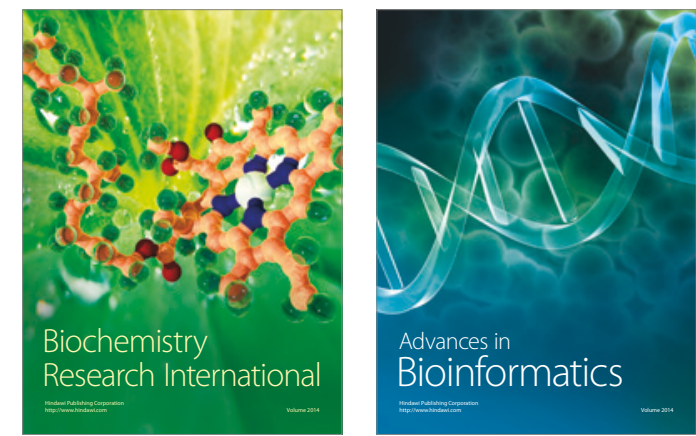

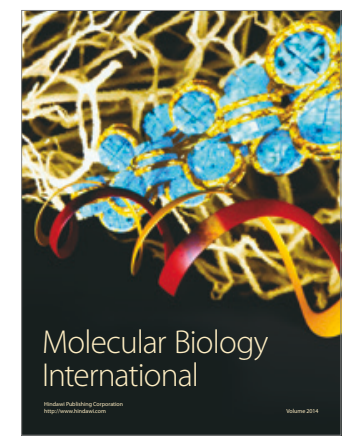

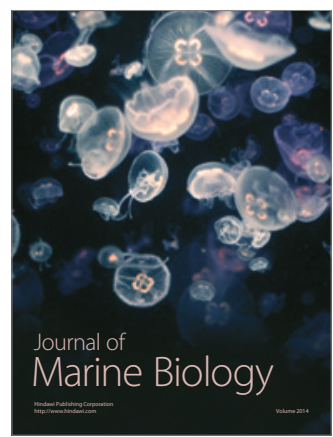

